\title{
Design Approach for the Development of a Cryomodule for Compact Crab Cavities for Hi-Lumi LHC
}

\author{
Shrikant Pattalwar ${ }^{\mathrm{a}, \mathrm{d}}$, Thomas Jones ${ }^{\mathrm{b}}$ Niklas Templeton ${ }^{\mathrm{b}}$, Philippe Goudket ${ }^{\mathrm{a}, \mathrm{d}}$, \\ Peter McIntosh $^{\mathrm{a}, \mathrm{d}}$, Alan Wheelhouse ${ }^{\mathrm{a}, \mathrm{d}}$, Graeme Burt ${ }^{\mathrm{c}, \mathrm{d}}$, Ben Hall ${ }^{\mathrm{c}, \mathrm{d}}$, \\ Loren Wright ${ }^{\mathrm{c}, \mathrm{e}}$ and Tom Peterson ${ }^{\mathrm{f}}$ \\ ${ }^{a}$ Accelerator Science and Technology Centre, STFC Daresbury Laboratory, Warrington WA44AD, UK \\ ${ }^{2}$ Technology Group, STFC Daresbury Laboratory, Warrington, WA44A, UK \\ ${ }^{c}$ University of Lancaster, Lancaster, $U K$ \\ ${ }^{d}$ Cockcroft Institute, STFC Daresbury Laboratory, Warrington, WA44AD, UK \\ ${ }^{e} T E-M S C-C, C E R N$, Geneva, Switzerland \\ ${ }^{f}$ Technical Division, Fermilab, P.O. Box 500, Batavia, IL 60510 USA
}

\begin{abstract}
A prototype Superconducting RF (SRF) cryomodule, comprising multiple compact crab cavities is foreseen to realise a local crab crossing scheme for the "Hi-Lumi LHC", a project launched by CERN to increase the luminosity performance of LHC. A cryomodule with two cavities will be initially installed and tested on the SPS drive accelerator at CERN to evaluate performance with high-intensity proton beams. A series of boundary conditions influence the design of the cryomodule prototype, arising from; the complexity of the cavity design, the requirement for multiple RF couplers, the close proximity to the second LHC beam pipe and the tight space constraints in the SPS and LHC tunnels. As a result, the design of the helium vessel and the cryomodule has become extremely challenging. This paper assesses some of the critical cryogenic and engineering design requirements and describes an optimised cryomodule solution for the evaluation tests on SPS.
\end{abstract}

Keywords: Cryomodule, HiLumi LHC, SRF Crab Cavities

PACS: $29.20 .-\mathrm{c}, 85.25 .-\mathrm{j}$

\section{INTRODUCTION}

In order to extend the discovery potential of LHC, CERN has launched a major upgrade program "Hi-LumiLHC" (High Luminosity LHC) ${ }^{1}$ to improve the luminosity performance of LHC, aiming for L $=5 \times 10^{34} \mathrm{~cm}^{-2} \mathrm{~s}^{-1}$ from 2022.
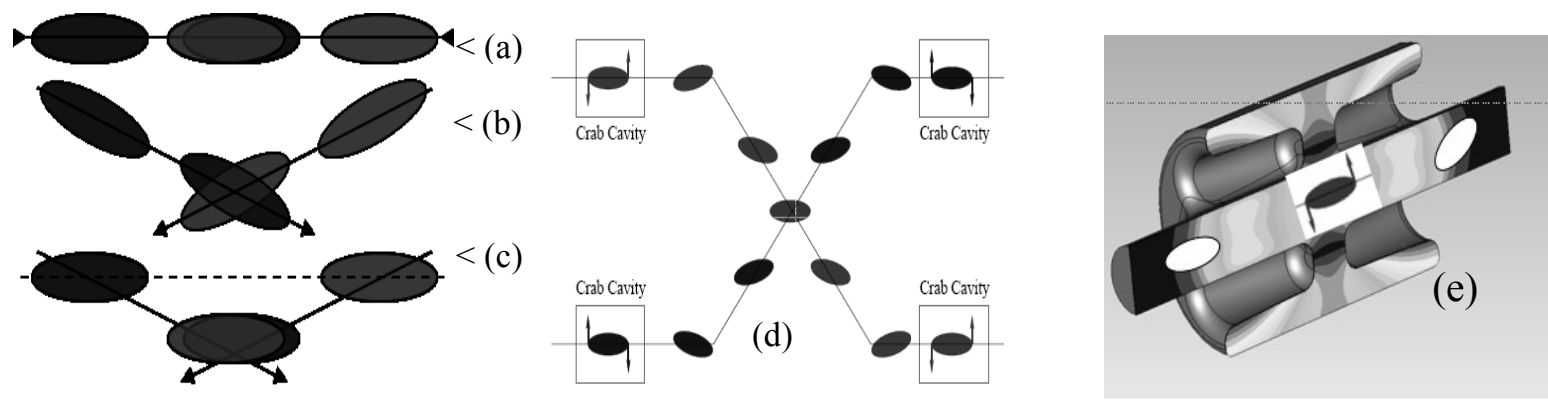

FIGURE 1. (a) Shows a head-on collision of the two particle bunches with maximum possible luminosity, (b) shows the existing collision configuration in LHC, (c) shows a concept of maximizing the luminosity by crabbing, (d) shows how crabbing can be achieved using a dipole field using SRF cavity (e) 
Luminosity or the number of collisions can be increased by using a crabbing technique as described in Figure 1. Such a technique has been demonstrated for electron beams at KEK but the associated technology that can be realised using SRF cavities, has never been utilised for hadron beams. Out of the several cavity designs proposed through a global effort, 3 cavity solutions have been chosen to carry forward for detailed studies. Table 1 summarise some of the key parameters ${ }^{2}$ specified for the Hi-Lumi LHC upgrade project.

Table 1. Summary of relevant beam parameters for HI-Lumi LHC upgrade.

\begin{tabular}{|c|c|c|c|}
\hline Parameter & Unit & Nominal & Upgrade \\
\hline Energy & $\mathrm{TeV}$ & \multicolumn{2}{|l|}{7} \\
\hline Protons/Bunch & $10^{11}$ & 1.15 & 1.7 \\
\hline Bunch Spacing & $\mathrm{nS}$ & \multicolumn{2}{|l|}{$50-25$} \\
\hline Crossing angle $\theta_{c}$ & $\mu \mathrm{rad}$ & 285 & $315-509$ \\
\hline Accelerating RF & $\mathrm{MHz}$ & \multicolumn{2}{|l|}{400} \\
\hline Crab RF & $\mathrm{MHz}$ & - & 400 \\
\hline $\begin{array}{l}\text { Peak Luminosity with Crab } \\
\text { Cavity }\end{array}$ & $10^{34} \mathrm{~cm}^{-2} \mathrm{~s}^{-1}$ & 1.0 & $7-10$ \\
\hline Pile up events per crossing & & 19 & $44-280$ \\
\hline
\end{tabular}

To mitigate any risks which may arise due to the complexity of the LHC it is necessary to understand the interaction between the powerful LHC beam and the RF crabbing field prior to its final implementation. Therefore a prototype SRF cryomodule, comprising of two compact crab cavities is foreseen to be installed and tested on the SPS drive accelerator at CERN prior to the long shut-down period LS2 to evaluate performance with high-intensity proton beams. A series of boundary conditions arising from the complexity of the cavity design, the requirement for multiple RF couplers, the close proximity of the cavity to the second LHC beam pipe and the tight space constraints in the SPS and LHC layouts influence the design of the cryomodule. As a result, the design of the helium vessel, the cryomodule and the overall integration with the SPS infrastructure have become extremely challenging.

This paper highlights the results of a preliminary study undertaken to assess some of the key cryogenic and engineering requirements and develop a design approach to develop a cryomodule for crab cavity tests with SPS.

\section{COMPACT CRAB CAVITIES}

A separation of only $194 \mathrm{~mm}$ between the two beam pipes in LHC is a major limitation and demands a compact cavity configuration. Three cavity designs, namely Dipole Cavity proposed by ODU and SLAC ${ }^{3}$, Double Quarter Wave by BNL ${ }^{4}$, and the 4-Rod ${ }^{5}$ by Lancaster University UK, have successfully overcome this basic limitation and are being prepared for the SPS tests. These cavity designs are physically very different, requiring a diverse array of mode coupler interfaces and configurations, thereby requiring three alternative cryomodule designs to accommodate. This paper is based on the cryomodule development for the 4-Rod cavity as shown in the Figure 2 . A proof of principle cavity has been fabricated and is ready for vertical tests at CERN. 

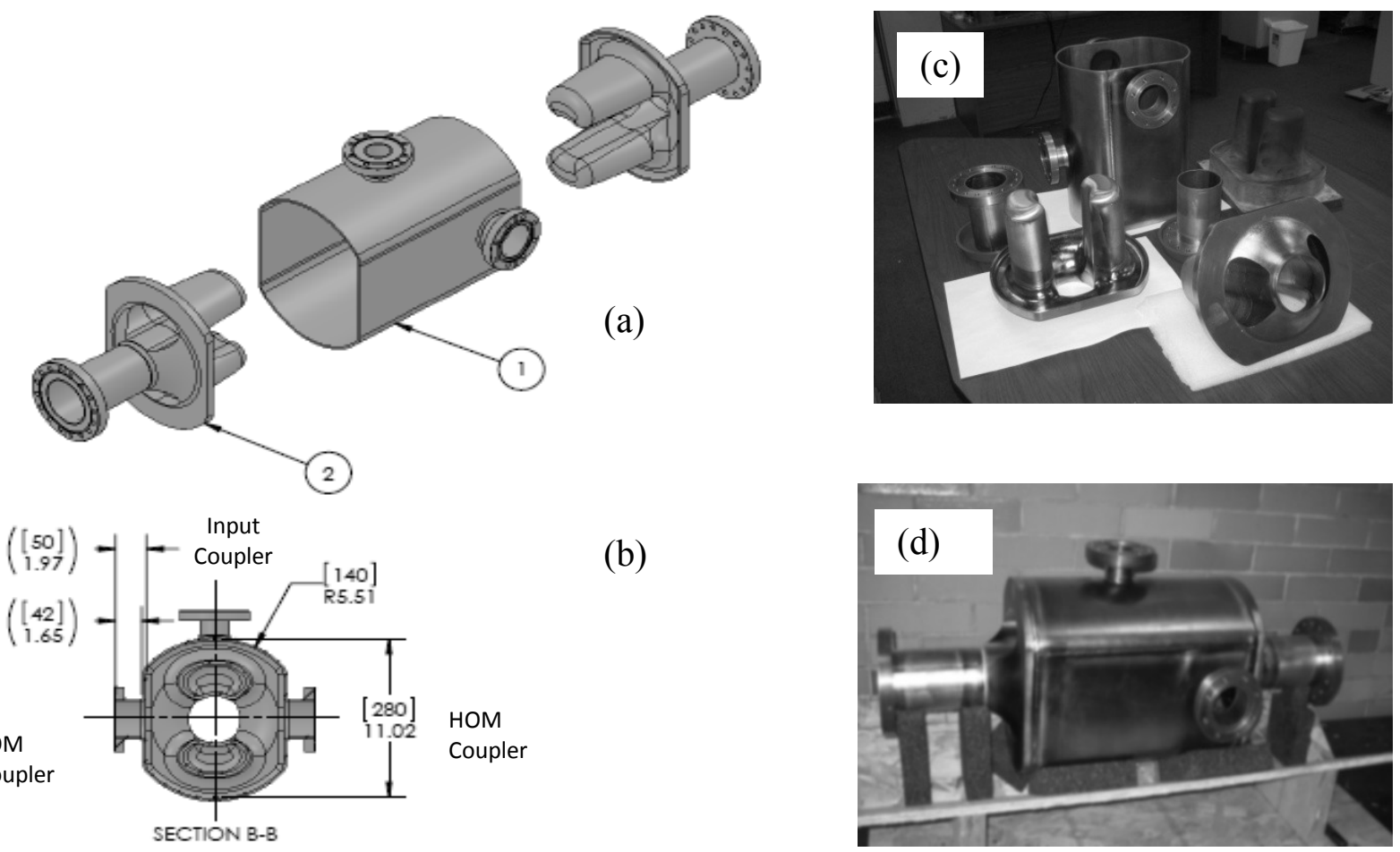

FIGURE 2. Details of the 4-Rod cavity (a) and (b) show the engineering drawings, (c) shows various parts of the cavity before EB welding (d) shows the completed cavity.

\section{REQUIREMENTS AND BOUNDARY CONDITIONS}

Various factors affecting the cryostat design arising from the boundary conditions improsed have been classified into 6 categories for the assessment as a part this study:

1. The crab cavity design - defines the operating temperature $(2 \mathrm{~K})$, limit of magnetic shielding $(<1 \mu \mathrm{T})$, types and orientation of the RF interfaces, tunability requirements and the dynamic power dissipition (heat load) to the liquid helium bath. ( 2.5W/ Cavity).

2. LHC - As the final goal is to implement the crabbing system on LHC it is necessary to consider the effect of the second beam pipe in the overall design and operation during the SPS tests. As a result, a dummy beam pipe will be included inside the helium vessel. In the final configuration on LHC the crabbing will be required for both the beams and it is desired that the cavity and the helium vessel are symmetrically located with respect to the two beam pipes.

Several alignment constrains arise due to transverse and longitudinal tolerances derived from LHC performance requirements, for example; minimum transverse rotation of $0.3^{\circ}$ per cavity, cavity tilt wrt the beam axis of 1 $\mathrm{mrad}$, tolerence in transverse plane of $0.7 \mathrm{~mm}$.

3. SPS Infrastructure - The SPS layout is more complex, mainly due to the Y section as shown in Figure 3 and the additional (default) vacuum pipe. The layout also defines the overall size restriction of the cryomodule and its interfaces for RF and cryogenic services. Additional restrictions relating to the limited accessibility to SPS, requires controlling the alignment of the cavities and the cryomdule with reference to beam via remote operation. In addition a series of integration issues ${ }^{6}$ will make these tests with SPS extremely challenging. 


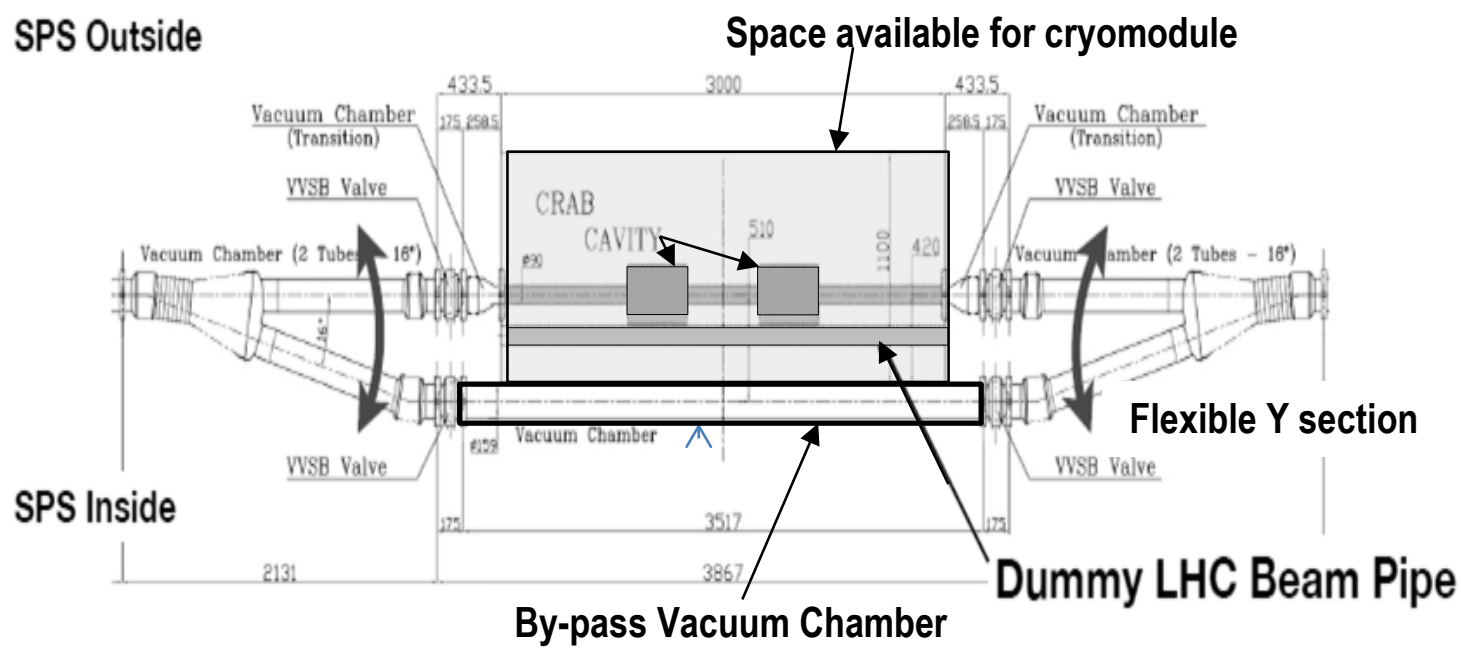

FIGURE 3. SPS layout with Y chamber

4. European PED (Pressure Enforcement Directive) ${ }^{7}-$ The cavities, the helium vessel and the cryomodule will undergo a series of cryogenic tests at SM18 at CERN, followed by complete system tests on SPS. Thermal and mechanical designs of the cavity, helium vessel and all associated components of the cryomodule must satisfy the PED requirements. In principle it is possible to design a cryomodule to the lowest category, however the lower limit of 1.8 bar (absolute) that must be assigned to the relief valves as set by SPS requirements ${ }^{8}$ may raise the PED category of the system to 1 or higher, as shown in Figure 4.
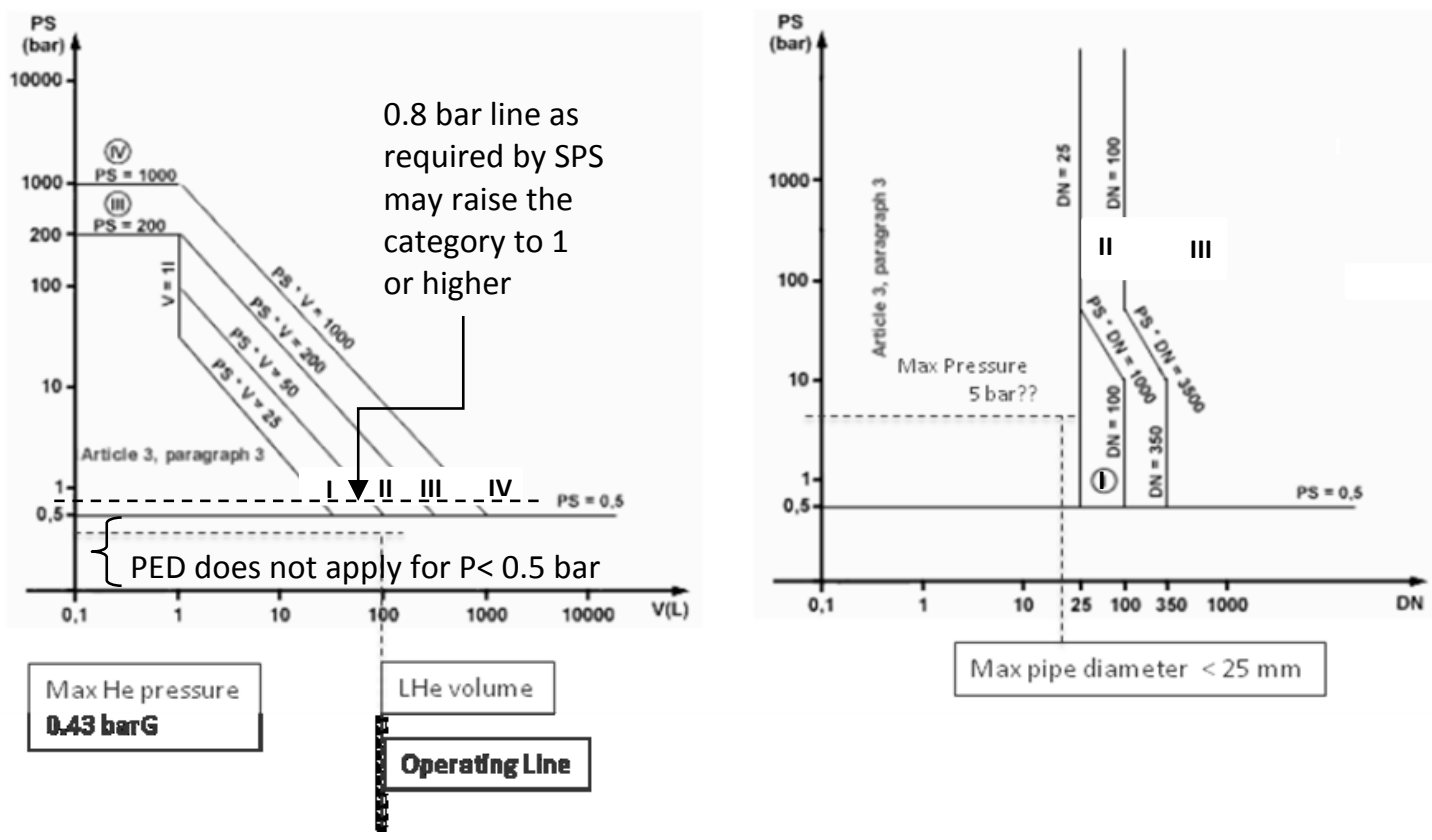

FIGURE 4. Categorising the Cryomdule as per European PED. In principle a cryomodule can be designed to limit the operation to 0.5 barA to exclude it from the regulation but raising the limit to 0.8 bar due to SPS requirements will put the design in PED category 1 or 2 depending upon the volume of liquid helium vessel. 
5. Cryogenic Services - The cooling power required to cool the cavities to $2 \mathrm{~K}$ will be supplied by a TCF-20 liquid helium liquefier in conjuction with warm vacuum pumps ${ }^{9}$. The cryo-plant is expected to provide approximately $15 \mathrm{~W}$ of cooling power at $2 \mathrm{~K}$. Although supplemental liquid helium will be provided from a storage dewar, the cryomodule should be designed to keep the total heat load budget near this limit. The space available at the SPS test-location also restricts the amount of helium inventory which in turn will limit the size of the helium vessel. In order to limit the cryomdule to PED category-1 it is anticipated that the liquid helium volume for each vessel will have to be less than 40 liters. Achieving this could be challenging as the inclusion of the second beam pipe inside the helium vessel will require additional space. Therefore this issue will have to be studied more carefully.

6. Other factors - There are several factors, conventional and unconventional, that must be taken into account in the design process; for example, schedule, costs, lessons learnt in the past ${ }^{10}$ and availability of relevant parts in the market etc.

\section{HELIUM VESSEL}

Taking into consideration all the above factors, a conceptual design of a helium vessel has been developed for the 4-Rod cavity as shown in Figure 5. All the necessary components of the helium vessel have been identified and modeled and initial mechanical and thermal analysis has been carried out, by choosing Titanium grade 5 as the material for the helium vessel but with an option to use stainless steel if deemed to be suitably appropriate. Some of the important features of the helium vessel assembly are described below.
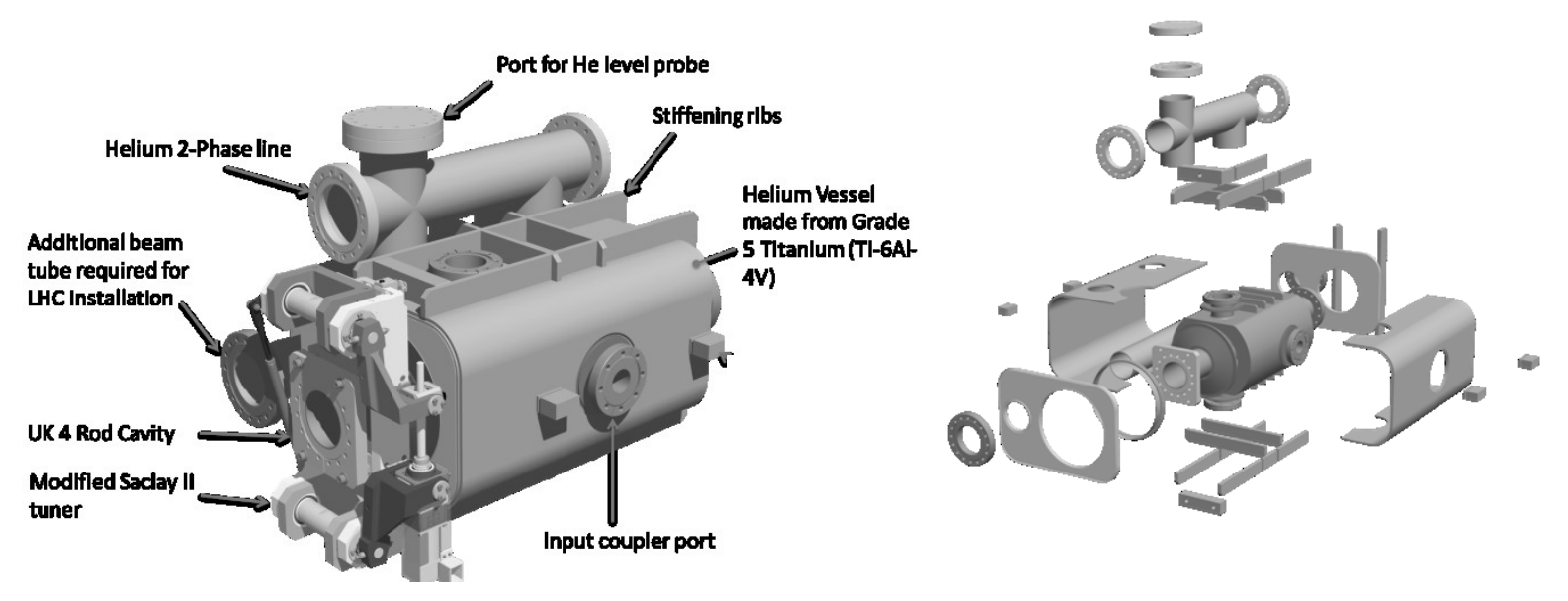

FIGURE 5. Conceptual design of a helium vessel (left) with all the parts for manufacturing (right)

1. A dummy beam pipe to represent the second LHC beam pipe is included in the helium vessel.

2. The helium vessel is completely filled with superfluid liquid helium and is attached to a two-phase line via two interfacing chimneys.

3. Provision is made to incorporate a modified Saclay type II tuner that has been successfully used on the ERL cryomodule for ALICE ${ }^{11}$.

4. The helium vessel has 3 penetrations for three RF coupler ports (Input, HOM and LOM) located in three different orientations (see Figure 2 above). At a differential pressure of 2 bar, the vessel experiences a maximum stress of $76 \mathrm{MPa}$, and a maximum deflection of $0.58 \mathrm{~mm}^{12}$. The cavity subsequently experiences a maximum stress of $60 \mathrm{MPa}^{13}$.

\section{CONCEPTUAL CRYOMODULE}

Figure 6 shows a conceptual design of a complete cryomodule consisting of two cavities that meets the primary geometrical requirements of the SPS tests ${ }^{14}$. The cryomodule is designed to give full access to internal parts. Such an approach simplifies the assembly procedure, requiring minimum tooling and thereby reducing the overall manufacturing costs. A novel approach ${ }^{15}$ of using a triple tube support assembly, with negligible displacement due to thermal contraction for the supporting the helium vessel has been incorporated as illustrated in Figure 7. 


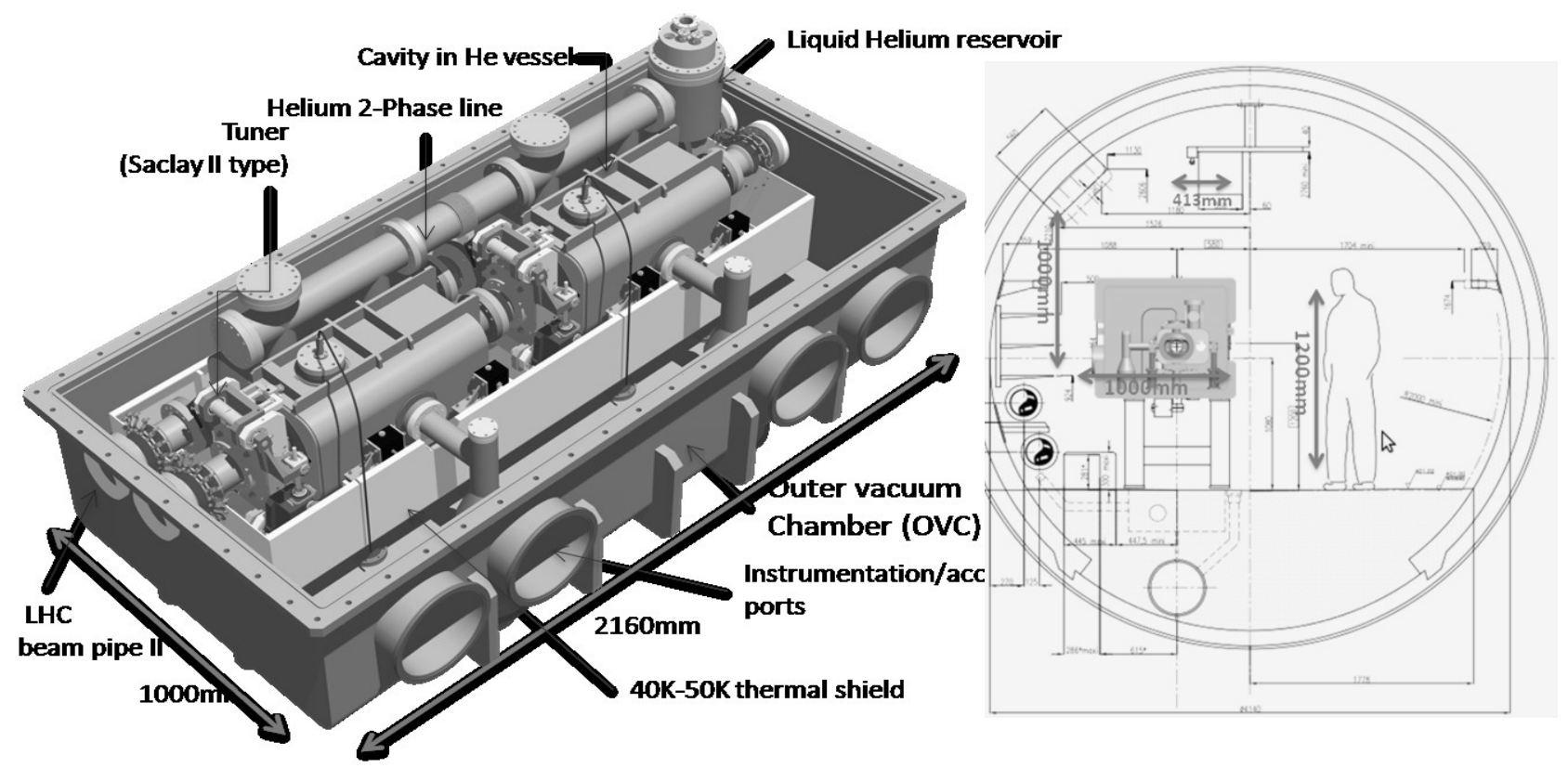

FIGURE 6. Concpetual design of a Cryomdule (left) and how it can be located in SPS (right)

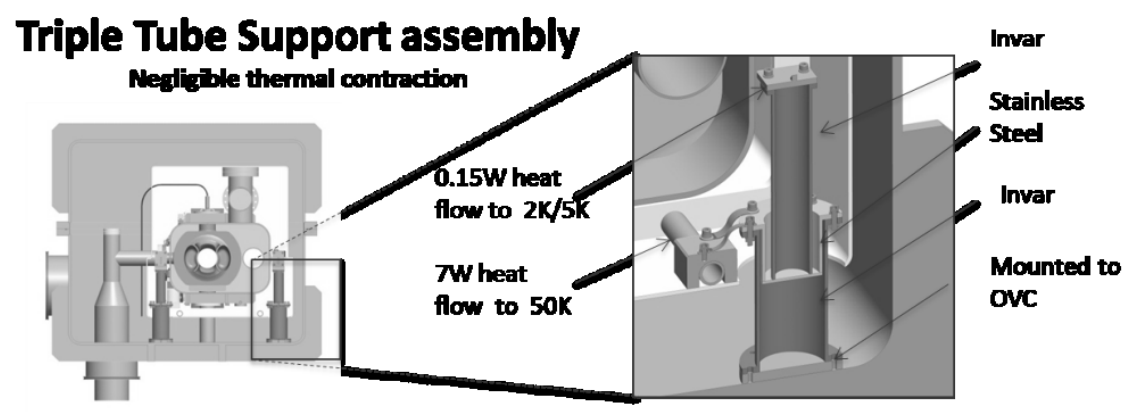

Triple Tube Contraction summary table

\begin{tabular}{|l|c|c|c|c|}
\hline & Invar Warm & Steel & Invar Cold & Assembly \\
\hline Warm Length $(\mathrm{mm})$ & $\mathbf{1 5 0}$ & $\mathbf{5 0}$ & $\mathbf{2 0 0}$ & $\mathbf{3 0 0}$ \\
\hline Temp. Range $(\mathrm{K})$ & $\mathbf{2 9 3 - 5 0}$ & $\mathbf{5 0 . 4 6 2}$ & $\mathbf{4 6 . 2 - 5}$ & $\mathbf{2 9 3 5}$ \\
\hline$\Delta L(\mathrm{~mm})$ & $\mathbf{0 . 0 3 2}$ & $\mathbf{0 . 1 6 7}$ & $\mathbf{0 . 0 8 0}$ & $\mathbf{- 0 . 0 3 5}$ \\
\hline Cold Length $(\mathrm{mm})$ & $\mathbf{1 0 9 . 9 6 8}$ & $\mathbf{4 9 . 5 5 3}$ & $\mathbf{1 9 9 . 9 2 0}$ & $\mathbf{3 0 0 . 0 3 4 7 5 6}$ \\
\hline
\end{tabular}

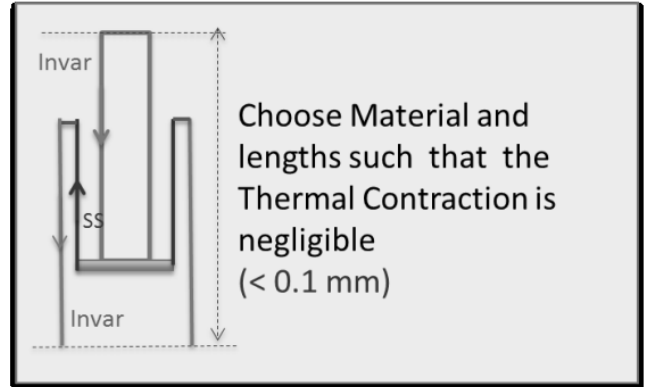

FIGURE 7. Each support consists of a triple tube assembly with negligible vertical displacement due to thermal contraction. Each cavity has 4 supports. 


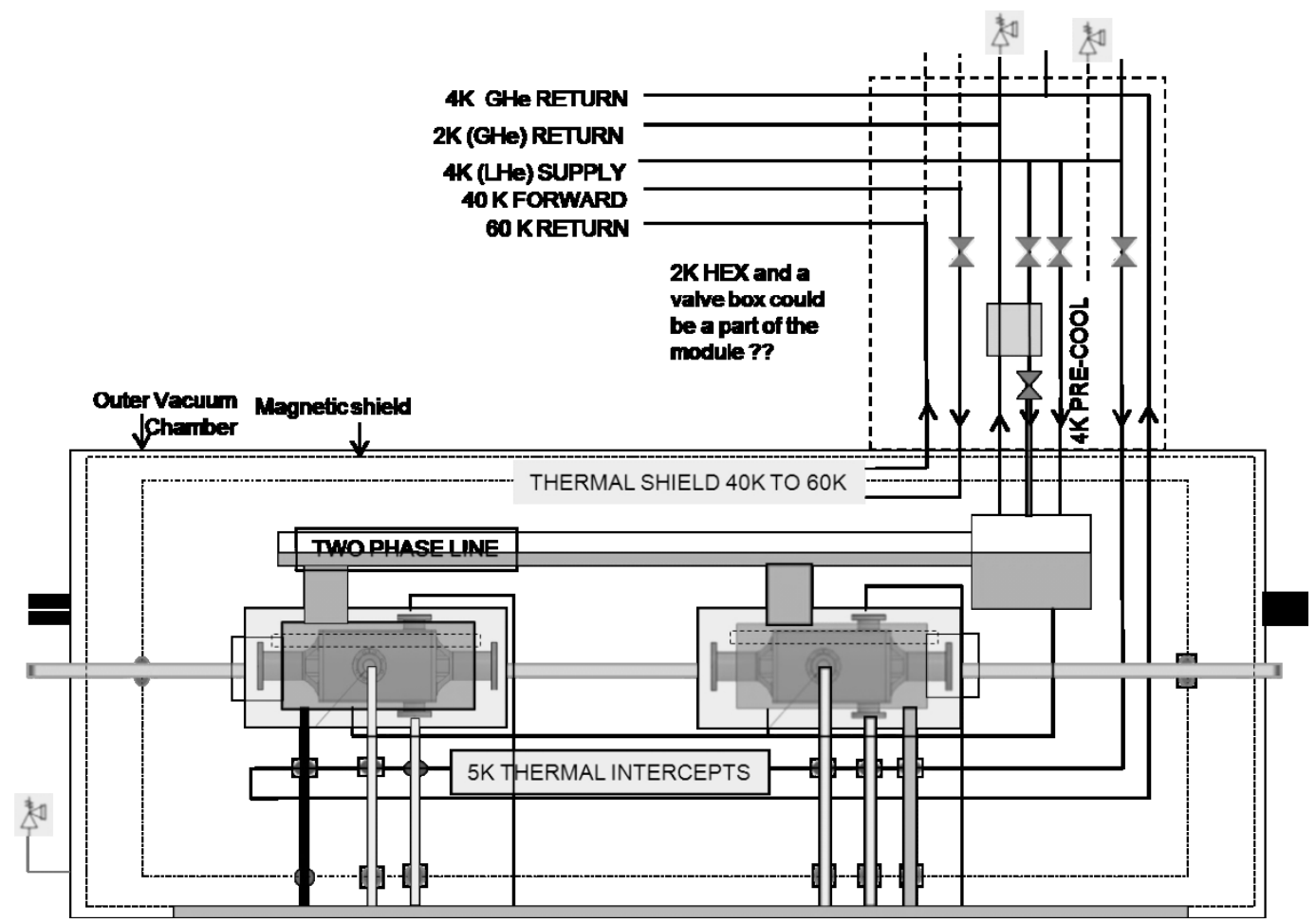

FIGURE 8. Schematic for cryogenic flow management inside the cryomodule.

Figure 8 shows a schematic for the cryogenic flow management of the cryomodule. Considering the limited capacity of the cryogenic infrastructure available at SPS, it will be necessary to avoid using the $4 \mathrm{~K}$ cooling circuit. Table 2 shows initial heat load estimates for the concept cryomodule.

Table 2. Heat load values in Watts estimated for the concept cryomdoule with two 4R cavities

\begin{tabular}{|c|c|c|c|}
\hline & $2 \mathrm{~K}$ & $4 \mathrm{~K}-6 \mathrm{~K}$ & $50 \mathrm{~K}-80 \mathrm{~K}$ \\
\hline \multicolumn{4}{|l|}{ Static } \\
\hline Radiation & 0.2 & 0.0 & 20.0 \\
\hline Supports & 0.1 & 1.2 & 57.2 \\
\hline RF-Couplers & 0.1 & 1.2 & 20.0 \\
\hline Tuners & 0.1 & & 5.0 \\
\hline Instrumentation & 0.1 & 0.1 & 1.0 \\
\hline Total Static & 0.6 & 2.5 & 103.2 \\
\hline \multicolumn{4}{|l|}{ Dynamic } \\
\hline RF Cavities & 5.0 & 0.0 & 0.0 \\
\hline RF Couplers & 0.2 & 4.0 & 40.0 \\
\hline Beam & 4.0 & 0.0 & 0.0 \\
\hline Total Dynamic & 9.2 & 4.0 & 40.0 \\
\hline Total Operating Load & 9.8 & 6.5 & 143.2 \\
\hline $\begin{array}{l}\text { Total Heat Load with a safety } \\
\text { margin of approx. } 1.5\end{array}$ & 15 & 10 & 215 \\
\hline
\end{tabular}




\section{SUMMARY AND CONCLUSION}

It is foreseen that the validation of the crab cavity system for providing optimum collision of high energy beam in the LHC, will require beam tests in the SPS involving two cavities in a horizontally configured cryostat. Three cavity designs are under consideration and it is anticipated that at least two designs will ultimately undergo tests in SPS in 2016 or 2017. Through a detail assessment and analysis, a conceptual design of a complete SRF cryomodule comprising two 4R cavities has been presented that meets all the primary requirements of the SPS tests. This work will be extended to optimize the design incorporating RF couplers, tuners and active alignment mechanism. This work is also anticipated to be extended to verify that the solution meets the detailed requirements of the SPS tests outlined in a specification document ${ }^{16}$ issued by CERN recently.

\section{ACKNOWLEDGMENTS}

This work has been undertaken as a part of The HiLumi LHC Design Study (a sub-system of HL-LHC) cofunded by the European Commission within the Framework Programme 7 Capacities Specific Programme, Grant Agreement 284404. Authors wish to acknowledge all the members of the WP4 of the HiLumi Collaboration and LARP for their valuable inputs and discussions.

\section{REFERENCES}

1. http://hilumilhc.web.cern.ch/hilumilhc/index.html

2. R. Calaga, E. Ciapala and E. Jensen, "Crab Cavities for the LHC Luminosity Upgrade", CERN project Document, (Aug 20, 2010)

3. J. Delayen, "Path to SPS testing of the prototype design of ODU/SLAC RF dipole cavity", Presentation at the Joint HiLumi LARP meeting NAPA, (April 8-10, 2013)

4. B. Xiao, "Proof of Principle cavity preparation and testing: DQW Cavity", Presentation at the Joint HiLumi LARP meeting NAPA, (April 8-10, 2013)

5. G. Burt, "UK 4R Cavity", Presentation at the LHC Crab cavity engineering meeting, Fermilab, (Dec 10-11, 2012)

6. A. Macpherson, "Cryostat issues for SPS crab cavity run: envelopes and interfaces", Presentation at the Crab Cavity Cryostat meeting, Fermilab, (May 30, 2013)

7. European Pressure Enforcement Directive http://ec.europa.eu/enterprise/sectors/pressure-and-gas/documents/ped/

8. O. Capatina, "General Specifications - LHC Crab Cavities", Presentation at the LHC Crab cavity engineering meeting, Fermilab, (Dec 10-11, 2012)

9. K. Brodzinski, "Cryogenic Circuits and Heat Loads", Presentation at the LHC Crab cavity engineering meeting, Fermilab, (Dec 10-11, 2012).

10. T.J. Peterson and J. Weisend II, "Introduction to Cryostat Design", A short course by Cryogenic Society of America, Tucson, (June 28, 2009)

11. P. A. McIntosh et al, "Realisation of a prototype superconducting CW Cavity and Cryomodule for Energy recovery", $S R F$ 2007, p545 (2007)

12. T. Jones, "Helium Vessel and Tuner for 4-Rod cavities", Presentation at the LHC Crab cavity engineering meeting, Fermilab, (Dec 10-11, 2012)

13. L. Alberty, "Prototype Cavity Stress Analysis", Presentation at the LHC Crab cavity engineering meeting, Fermilab, (Dec 10-11, 2012)

14. S. Pattalwar, "Cryostat Concepts 4-Rod Cavities", Presentation at the LHC Crab cavity engineering meeting, Fermilab, (Dec $10-11,2012)$

15. R. Done, "Pressure Cell Support System, form Fundamentals of cryogenics", STFC Cryogenics training, a lecture series, at DTFC, Daresbury Laboratory, (April 20, 2010).

16. P. Baudrenghien et al, "Functional Specifications of the LHC Prototype Crab Cavity System", http://cds.cern.ch/search?p=CERN-ACC-NOTE-2013-003, (Feb 28, 2013) 